\title{
A Characterization of Uninorms by Means of a Pre-order they Induce
}

\author{
Dana Hliněná ${ }^{a}$ and Martin Kalina ${ }^{b}$ \\ ${ }^{a}$ Department of Mathematics \\ Faculty of Electrical Engineering and Communication, Brno University of Technology \\ Technická 8, Cz-616 00 Brno, Czech Republic \\ hlinena@feec.vutbr.cz \\ ${ }^{b}$ Department of Mathematics and Descriptive Geometry \\ Faculty of Civil Engineering, Slovak University of Technology \\ Radlinského 11, Sk-810 05 Bratislava, Slovakia \\ kalina@math.sk
}

\begin{abstract}
In Hliněná et al. (2014) the authors, inspired by Karaçal and Kesicioğlu (2011), introduced a pre-order induced by uninorms. This contribution is devoted to a classification of families of uninorms by means of types of preorders (and orders) they induce. Philosophically, the paper follows the original idea of Clifford (1954).

Keywords: Pre-order induced by uninorm, Representable uninorm, Uninorm, Uninorm with continuous underlying operations, Locally internal uninorm.
\end{abstract}

\section{Introduction}

In this paper we study pre-orders generated by uninorms. The main idea is based on that of Karaçal and Kesicioğlu [19], and follows the original idea of Clifford [4]. The main idea of authors is to show a relationship between families of uninorms and families of pre-orders (partial orders, in some cases) they induce (see [16]). In some sense, the pre-order (see Definion 11) follows the original idea by Clifford [4]. Another relation induced by uninorms, that is always a partial order (see Definition 12), was proposed by Erteğrul et al. [11]. Here, the main intention of authors was to get a partial order. But this relation (partial order) does not extend the relation introduced by Clifford [4].

\section{Preliminaries}

In this section we review some well-known types of monotone commutative monoidal operations on $[0,1]$ and provide an overview of, from the point of view of this contribution, important steps in introducing orders (and pre-orders) induced by semigroups.

\subsection{Known types of monotone commutative monoidal operations on $[0,1]$}

In this part we give just very brief review of well-known types of monotone commutative monoidal operations on $[0,1]$. For more details we recommend monographs $[2,20]$.

Definition 1 (see, e.g., [20]). A triangular norm $T$ ( $t$ norm for short) is a commutative, associative, monotone binary operation on the unit interval $[0,1]$, fulfilling the boundary condition $T(x, 1)=x$, for all $x \in[0,1]$.

Definition 2 (see, e.g., [20]). A triangular conorm $S$ (t-conorm for short) is a commutative, associative, monotone binary operation on the unit interval $[0,1]$, fulfilling the boundary condition $S(x, 0)=x$, for all $x \in[0,1]$.

Remark 1. If $T$ is a t-norm, then

$$
S(x, y)=1-T(1-x, 1-y)
$$

is a t-conorm and vice versa. We obtain a dual pair $(T, S)$ of a t-norm and a t-conorm.

Example 1. Well-known examples of triangular norms and their dual t-conorms are:

- $T_{M}(x, y)=\min (x, y), \quad S_{M}(x, y)=\max (x, y)$,

- $T_{P}(x, y)=x . y, \quad S_{P}(x, y)=x+y-x . y$,

- $T_{L}(x, y)=\max (x+y-1,0), \quad S_{L}(x, y)=\min (x+$ $y, 1)$.

Casasnovas, Mayor [3] introduced divisible t-norms.

Definition 3 ([3]). Let $L$ be a bounded lattice and $T: L \times L \rightarrow L$ be a t-norm. $T$ is said to be divisible if the following conditions are satisfied for all $(x, y) \in L^{2}$

$$
(x \leq y) \Rightarrow(\exists z \in L)(T(y, z)=x) .
$$

Of course, a t-norm $T:[0,1]^{2} \rightarrow[0,1]$ is divisible if and only if it is continuous. 
Definition 4 (see, e.g., [2]). Let $*:[0,1]^{2} \rightarrow[0,1]$ be a binary commutative operation. Then

(i) element $c$ is said to be idempotent if $c * c=c$,

(ii) element $e$ is said to be neutral if $e * x=x$ for all $x \in L$,

(iii) element $a$ is said to be annihilator if $a * x=a$ for all $x \in L$.

Definition 5 ([26]). A uninorm $U$ is a function $U$ : $[0,1]^{2} \rightarrow[0,1]$ that is increasing, commutative, associative and has a neutral element $e \in[0,1]$.

Remark 2. For any uninorm with neutral element equal to $e$ we denote

$$
A(e)=[0, e[\times] e, 1] \cup] e, 1] \times[0, e[.
$$

1. If $e \notin\{0,1\}$ is the neutral element of $U$, we say that $U$ is a proper uninorm.

2. Every uninorm $U$ has a distinguished element $a$ called annihilator, for which the following holds $U(a, x)=U(0,1)=a$. A uninorm $U$ is said to be conjunctive if $U(x, 0)=0$, and $U$ is said to be disjunctive if $U(1, x)=1$, for all $x \in[0,1]$.

Lemma 1 ([12]). Let $U$ be a uninorm with the neutral element e. Then, for $(x, y) \in[0,1]^{2}$ the following holds

(i) $T(x, y)=\frac{U(e x, e y)}{e} \quad$ is a t-norm,

(ii) $S(x, y)=\frac{U((1-e) x+e,(1-e) y+e)-e}{1-e}$ is a t-conorm.

For all $(x, y) \in A(e)$ we have

$$
\min (x, y) \leq U(x, y) \leq \max (x, y) .
$$

Definition 6. Let $U$ be a uninorm. We say that $U$ is internal if $U(x, y) \in\{x, y\}$ for all $(x, y) \in[0,1]^{2}$.

$A$ uninorm $U$ is locally internal on a set $G \subset[0,1]^{2}$ if $U(x, y) \in\{x, y\}$ for all $(x, y) \in G$.

Remark 3. (a) Particularly, a uninorm $U$ is locally internal on the boundary if $U(x, 0) \in\{x, 0\}$ and $U(x, 1) \in\{x, 1\}$ holds for all $x \in[0,1]$. Some examples of uninorms which are not locally internal on the boundary can be found, e.g., in $[14,15,16]$, see also Fig. 1.

(b) An important family of uninorms is that of internal ones. From results by Drewniak and Drygaś [6] follows that the family of all internal uninorms is identical with that of idempotent uninorms. Some further study of locally internal uninorms can be found, e.g., in [8] and in literature referenced therein.

From results in $[6,21,25]$ we have the following.

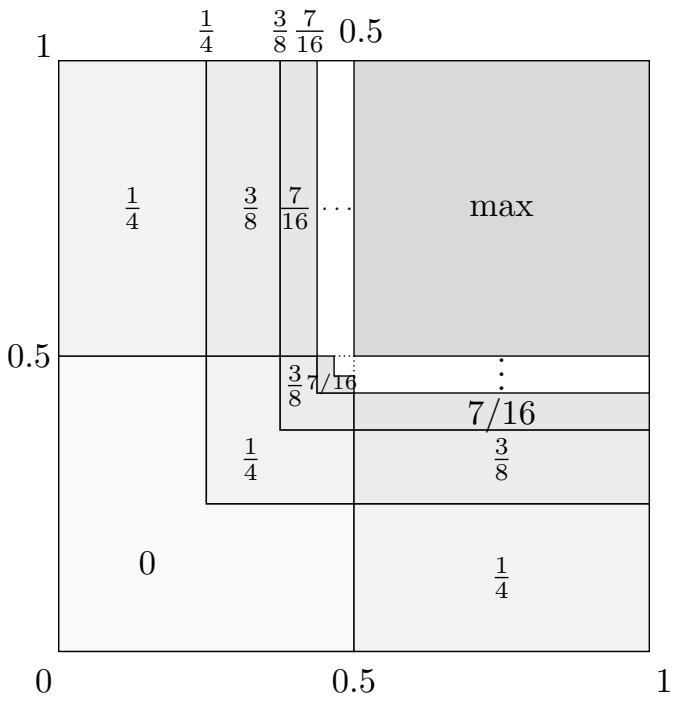

Figure 1: Example of a uninorm not locally internal on the boundary (the L-shaped areas of constantness being right-side closed)

Lemma 2. Let $U$ be a uninorm. $U$ is idempotent if and only if it is $U$ internal.

Proposition 1 (E.g., [10]). Let $f:[-\infty, \infty] \rightarrow[0,1]$ be an increasing bijection. Then

$$
U(x, y)=f^{-1}(f(x)+f(y))
$$

is a uninorm that is continuous everywhere except at points $(0,1)$ and $(1,0)$, and is strictly increasing on ] $0,1\left[^{2} . U\right.$ is conjunctive if we adopt the convention $-\infty+\infty=-\infty$, and $U$ is disjunctive adopting the convention $-\infty+\infty=\infty$.

Definition 7 (E.g., [10]). The uninorm $U$ fulfilling formula (2) for an increasing bijection $f:[-\infty, \infty] \rightarrow$ $[0,1]$ adopting either of the conventions, $-\infty+\infty=$ $-\infty$ or $-\infty+\infty=\infty$, is said to be a representable uninorm.

Remark 4. Representable uninorms, under the name aggregative operators were studied already by Dombi [5].

Another important class of uninorms is that of continuous ones on $] 0,1[2$. These uninorms were characterized by $\mathrm{Hu}$ and $\mathrm{Li}$ [17], and further studied by Drygaś [7]. From results in [17] we have the following characterization.

Proposition 2. A uninorm $U$ with neutral element $e \in] 0,1[$ is continuous on on $] 0,1]^{2}$ if and only if one of the following conditions is satisfied:

(i) $U$ is representable,

(ii) there exists $0<a<e$, a continuous t-norm $T$ a representable uninorm $U_{r}$ and an increasing bijection $\varphi:[a, 1] \rightarrow[0,1]$ such that 
$U(x, y)=\varphi^{-1}\left(U_{r}(\varphi(x), \varphi(y))\right)$ for $(x, y) \quad \in$

$[a, 1]^{2}$,

$U(x, y)=a T\left(\frac{x}{a}, \frac{y}{a}\right)$ for $(x, y) \in[0, a]^{2}$,

$U(x, y)=\min \{x, y\}$ for $(x, y) \in[0, a[\cap] a, 1[\cup$

]$a, 1[\cap[0, a[$,

and $U$ is locally internal on the boundary,

(iii) or there exists $e<b<1$ a continuous t-conorm $S$ and a representable uninorm $U_{r}$ and an increasing bijection $\varphi:[0, b] \rightarrow[0,1]$ such that

$U(x, y)=\varphi^{-1}\left(U_{r}(\varphi(x), \varphi(y))\right)$ for $(x, y) \in[0, b]^{2}$, $U(x, y)=b+(1-b) S\left(\frac{x-b}{1-b}, \frac{y-b}{1-b}\right)$ for $(x, y) \in[b, 1]^{2}$, $U(x, y)=\max \{x, y\}$ for $(x, y) \in] b, 1] \cap[0 . b[\cup$ $[0, b[\cap] b, 1]$,

and $U$ is locally internal on the boundary.

\subsection{An overview of pre-orders induced by a semigroup}

The study of orders (pre-orders) induced by a semigroup operation had started by Clifford [4]. Later, Hartwig [13] and independently also Nambooripad [23], defined a partial order on regular semigroups. Their definition is the following.

Definition $8([13,23])$. Let $(S, \oplus)$ be a semigroup and $E_{S}$ the set of its idempotent elements. Then

$$
a \leq_{\oplus} b \Leftrightarrow\left(\exists e, f \in E_{S}\right)(a=b \oplus e=f \oplus b) .
$$

If the relation $\leq_{\oplus}$ is a partial order on $S$, it is called natural.

Definition 8 was generalized by Mitch [22].

Definition $9([22])$. Let $(S, \oplus)$ be an arbitrary semigroup. By $\leq_{\oplus}$ we denote the following relation

$$
a \leq_{\oplus} b \quad \Leftrightarrow \quad a=b \oplus z_{1}=z_{2} \oplus b, a \oplus z_{1}=a
$$

for some $z_{1}, z_{2} \in E_{S^{1}}$, where

$$
S^{1}= \begin{cases}S & \text { if } S \text { has a neutral element, } \\ S \cup\{e\} & \text { otherwise, where e plays } \\ \text { the role of the neutral element, }\end{cases}
$$

and $E_{S^{1}}$ is the set of all idempotents of $S^{1}$.

Lemma $3([22])$. Let $(S, \oplus)$ be an arbitrary semigroup. The relation $\leq_{\oplus}$ is reflexive and antisymmetric on $S$.

Proposition $3([22])$. Let $(S, \oplus)$ be an arbitrary semigroup. The relation

$$
a \lesssim \oplus b a=x \oplus b=b \oplus y
$$

for some $x, y \in S^{1}$, is a partial order on $S$.

From now on, we restrict our attention to commutative semigroups. Lemma 3 and Proposition 3 immediately imply the following.
Lemma 4. Let $(S, \oplus)$ be a commutative semigroup. By $a_{\lesssim \oplus}$ we denote the set

$$
a_{{ }_{\oplus}}=\{z \in S ; z \lesssim \oplus a\},
$$

where $a \in S$. Then for all $a, b \in S$ it holds that $a \lesssim_{\oplus} b$ if and only if $a_{{ }_{\oplus}} \subseteq b_{{ }_{\oplus}}$.

Directly by Definition 9 we get

Proposition 4. Let $(S, \oplus)$ be a commutative semigroup. Then the set $a_{{ }_{\oplus}}$ is an ideal in $(S, \oplus)$.

Lemma 5. Let $(S, \oplus)$ be a commutative semigroup. Let $I_{S}$ be an ideal of $(S, \oplus)$. Then $\left(I_{S}, \oplus_{I_{S}}\right)$ is a subsemigrup of $(S, \oplus)$, where $\oplus_{I_{S}}=\oplus \uparrow I_{S}^{2}$.

Karaçal and Kesicioğlu [19] defined a partial order on bounded lattices $L$ by means of t-norms.

Definition 10 ([19]). Let $L$ be a bounded lattice and $T: L \times L \rightarrow L$ a t-norm. We write $x \preceq_{T} y$ for arbitrary $x, y \in L$, if there exists $z \in L$ such that $x=$ $T(y, z)$.

Proposition 5 ([19]). Let $L$ be a bounded lattice and $T: L \times L \rightarrow L$ a t-norm. Then the relation $\preceq_{T}$ is a partial order on $L$.

Remark 5. For arbitrary t-norm $T$, the partial order $\preceq_{T}$ from Definition 10 extends the partial order $\lesssim_{T}$ from Definition 9 in the following sense:

let $L$ be arbitrary bounded lattice and $T$ a commutative semigroup-operation on $L$ with a neutral element such that $\left(L, \preceq_{T}\right)$ is a partially ordered set. Then

$$
a \lesssim_{T} b \Rightarrow a \preceq_{T} b
$$

for all $a, b \in L$.

Remark 6. Concerning a correspondence between properties of binary aggregation function $A: L^{2} \rightarrow L$ and relation $\preceq_{A}$ (changing a t-norm $T$ for $A$ in Definition 10), the following can be said:

- if $A$ has a neutral element, or $A$ is idempotent, then $\preceq_{A}$ is reflexive,

- if $A$ is associative, then $\preceq_{A}$ is transitive,

- the anti-symmetry of $\preceq_{A}$ fails if there exist elements $x \neq z$ and $y_{1}, y_{2}$ such that $z=A\left(x, y_{1}\right)$ and $x=A\left(z, y_{2}\right)$. Hence, if one of the following

$$
\begin{aligned}
& x \preceq_{A} z \quad \Rightarrow \quad x \leq_{L} z, \\
& x \preceq_{A} z \quad \Rightarrow \quad z \leq_{L} x
\end{aligned}
$$

holds then $\preceq_{A}$ is anti-symmetric.

Hliněná et al. [16] introduced the following relation $\preceq_{U}$. 
Definition 11 ([16]). Let $U:[0,1]^{2} \rightarrow[0,1]$ be an arbitrary uninorm. $B y \preceq_{U}$ we denote the following relation

$x \preceq_{U} y$ if there exists $\ell \in[0,1]$ such that $U(y, \ell)=x$.

Immediately by Definition 11 we get the next lemma.

Lemma 6. Let $U$ be an arbitrary uninorm. Then $\preceq_{U}$ is transitive and reflexive. If $a$ and $e$ are the annihilator and the neutral elements of $U$, respectively, then

$$
a \preceq_{U} x \preceq_{U} e
$$

holds for all $x \in[0,1]$.

Remark 7. In Definition 11 we have used the same notation $\preceq_{U}$ for the pre-order defined from a uninorm $U$, as in Definition 10 for the corresponding partial order $\preceq_{T}$ defined from a t-norm $T$. These two relations really coincide if $U=T$, i.e., the notation should not cause any problems.

The pre-order $\preceq_{U}$ extends the partial order $\lesssim_{U}$ from Definition 9 in the following sense.

Proposition 6. Let $U$ be an arbitrary uninorm. Then

$$
x \lesssim_{U} y \Rightarrow x \preceq_{U} y
$$

for all $(x, y) \in[0,1]^{2}$.

A different type of partial order induced by uninorms has been defined by Ertuğrul et al. [11].

Definition 12 ([11]). Let $U$ be a uninorm and $e \in$ ] $0,1\left[\right.$ its neutral element. For $(x, y) \in[0,1]^{2}$ denote $x \unlhd_{U} y$ if one of the following properties is satisfied:

1. there exists $\ell \in[0, e]$ such that $x=U(y, \ell)$ and $(x, y) \in[0, e]^{2}$,

2. there exists $\ell \in[e, 1]$ such that $y=U(x, \ell)$ and $(x, y) \in[e, 1]^{2}$,

3. $0 \leq x \leq e \leq y \leq 1$.

Proposition 7 ([11]). For an arbitrary uninorm $U$, the relation $\unlhd_{U}$ from Definition 12 is a partial order.

Example 2. Consider the following uninorm $U$

$$
U(x, y)= \begin{cases}\min (x, y) & \text { if }(x, y) \in\left[0, \frac{1}{2}\right]^{2} \\ \max (x, y) & \text { otherwise }\end{cases}
$$

Then $\unlhd_{U}$ coincides with the usual order of $[0,1]$, while $x \lesssim_{U} y$ if one of the following possibilities is satisfied

- $y \leq x$ for $x>0.5$

- $x \leq y$ for $(x, y) \in\left[0,0.5\left[^{2}\right.\right.$,
- $y=0.5$.

Remark 8. Let $U$ be a uninorm. To compare the relation $\preceq_{U}$ from Definition 11 with $\unlhd_{U}$ from Definition 12 , the following should be remarked.

(i) The relation $\preceq_{U}$, given in Definition 11 is a preorder, but not necessarily a partial order. Unlike this, the relation $\unlhd_{U}$ defined by Definition 12 , is always a partial order.

(ii) As illustrated by Example 2, the partial order $\unlhd_{U}$ does not necessarily extends the partial order $\lesssim_{U}$ on the semigroup $([0,1], U)$, i.e.,

$$
x \lesssim_{U} y \nRightarrow x \unlhd_{U} y .
$$

As shown by Proposition 6 , the pre-order $\preceq_{U}$ always extends the partial order $\lesssim_{U}$ on $([0,1], U)$, see formula (4).

Further in the text, we will consider only the pre-order $\preceq_{U}$ to distinguish several families of uninorms.

Definition 13. Let $U$ be an arbitrary uninorm.

(i) For $(x, y) \in[0,1]^{2}$ we denote $x \sim_{U} y$ if $x \preceq_{U} y$ and $y \preceq_{U} x$.

(ii) For $(x, y) \in[0,1]^{2}$ we denote $x \|_{U} y$ if neither $x \preceq_{U}$ y nor $y \preceq_{U} x$ holds, and $d x \nVdash_{U} y$ if $x \preceq_{U}$ y or $y \preceq_{U} x$.

(iii) For arbitrary $x \in[0,1]$ we denote $x_{\sim_{U}}=\{z \in$ $\left.[0,1] ; z \sim_{U} x\right\}$.

\section{Some distinguished families of uninorms and properties of the corresponding pre-orders}

We are going to study a relationship between some distinguished families $\mathcal{U}$ of uninorms on the one hand and properties of the corresponding pre-orders $\preceq_{U}$ for $U \in \mathcal{U}$ on the other hand.

A direct consequence to Lemma 6 is the following.

Corollary 1. Let $U$ be a uninorm. The following holds for all $x \in[0,1]$ :

(i) $0 \preceq_{U} x$ if and only if $U$ is conjunctive,

(ii) $1 \preceq_{U} x$ if and only if $U$ is disjunctive.

\subsection{Locally internal uninorms}

In this part we distinguish three types of locally internal uninorms:

- on the boundary,

- on $A(e)$

- on $[0, e]^{2} \cup[e, 1]^{2}$. 
Proposition 8. Let $U$ be a uninorm. It is locally internal on the boundary if and only if for every element $x \in[0,1]$

$$
0 \sharp_{U} x \text { and } 1 \sharp_{U} x \text {. }
$$

Proposition 9. Let $U$ be a uninorm with neutral element $e$. It is locally internal on $A(e)$ if and only if $\preceq_{U}$ is a partial order and for every element $x \in[0, e]$ and $y \in[e, 1]$ we have

$$
x \nVdash_{U} y \text {. }
$$

Remark 9. For an arbitrary uninorm $U$ and for a pair $(x, y) \in[0,1]^{2}$, we have

$$
\begin{aligned}
& U(x, y)=x \quad \Rightarrow \quad x \preceq_{U} y, \\
& U(x, y)=y \quad \Rightarrow \quad y \preceq_{U} x .
\end{aligned}
$$

Results in [8] imply that if a uninorm $U$ is locally internal on $A(e)$, there are three possibilities:

(a) $U(x, y)=\min \{x, y\}$ for all $(x, y) \in A(e)$,

(b) $U(x, y)=\max \{x, y\}$ for all $(x, y) \in A(e)$,

(c) there exists a (not necessarily strictly) decreasing function $f:[0, e[\rightarrow] e, 1]$ such that, for $(x, y) \in$ $[0, e[\times] e, 1]$, we have

$$
U(x, y) \begin{cases}=x & \text { if } y<f(x) \\ =y & \text { if } y>f(x) \\ \in\{x, y\} & \text { if } y=f(x)\end{cases}
$$

Proposition 10. Let $U$ be a uninorm. If $U$ is locally internal on $[0, e]^{2} \cup[e, 1]^{2}$, then $\preceq_{U}$ is a linear order.

Example 3. Let $U:[0,1]^{2} \rightarrow[0,1]$ be defined by

$$
U(x, y)= \begin{cases}x y & \text { if } \max \{x, y\} \leq 0.6 \\ \min \{1, x+y\} & \text { if } \min \{x, y\} \geq 0.6 \\ \min \{x, y\} & \text { otherwise }\end{cases}
$$

Then $U$ is a uniform with its neutral element $e=0.6$ and annihilator $a=0$. $U$ generates the following relation $\preceq_{U}$

$$
x \preceq_{U} y \Leftrightarrow \begin{cases}x \leq y & \text { and } x<0.6 \\ x \geq y & \text { and } x, y \geq 0.6 .\end{cases}
$$

The uninorm $U$ is just locally internal on $A(e)$ and not internal, but $\preceq_{U}$ is a linear order.

\subsection{Uninorms with continuous underlying t-norm and t-conorm}

Results in [19] imply the following.
Proposition 11. Let $U$ be a proper uninorm with a neutral element e. Then $U$ has continuous underlying t-norm and t-conorm if and only if the following hold

$$
\begin{aligned}
& x \leq y \Rightarrow x \preceq_{U} y \quad \text { for }(x, y) \in[0, e]^{2}, \\
& y \leq x \Rightarrow x \preceq_{U} y \quad \text { for }(x, y) \in[e, 1]^{2} .
\end{aligned}
$$

Propositions 9 and 11 have the following corollary.

Corollary 2. Let $U$ be a proper uninorm. Then $U$ is locally internal on $A(e)$ and with continuous underlying $t$-norm and $t$-conorm if and only if $\preceq_{U}$ is a linear order.

Applying Proposition 2 to the pre-order $\preceq_{U}$ we get the following characterization of representable uninorms.

Proposition 12. A uninorm $U$ is representable if and only if for all $(x, y) \in] 0,1\left[^{2}\right.$ we have $x \sim_{U} y$.

Proposition 2 implies the following characterization of uninorms continuous on $] 0,1\left[^{2}\right.$.

Proposition 13. Let $U$ be a proper uninorm with neutral element $e$, which is not representable. Then it is continuous on $] 0,1\left[^{2}\right.$ if and only if one of the following is valid.

(i) There exists $0<a<e$ such that

$$
\begin{aligned}
& \text { 1. } \left.x \sim_{U} y \text { for all }(x, y) \in\right] a, 1\left[^{2},\right. \\
& \text { 2. } x \preceq_{U} y \Leftrightarrow x \leq y \text { for all }(x, y) \in[0, a]^{2} .
\end{aligned}
$$

(ii) There exists $e<b<1$ such that

$$
\begin{aligned}
& \text { 1. } \left.x \sim_{U} y \text { for all }(x, y) \in\right] 0, b\left[^{2},\right. \\
& \text { 2. } x \preceq_{U} y \Leftrightarrow x \geq y \text { for all }(x, y) \in[b, 1]^{2} .
\end{aligned}
$$

\subsection{Some other classes of uninorms on $[0,1]$}

First, we provide some results on uninorms with an area of constantness in $[0, e]^{2}$ or $[e, 1]^{2}$.

Proposition 14 ([14]). Let $U$ be a proper uninorm having $e$ as neutral element. Let $y>e$ be an idempotent element of $U$. If there exists $x<e$ such that $U(x, y)=\tilde{x} \in] x, e[$ then

$$
U(z, y)=\tilde{x} \text { and } U(z, x)=U(\tilde{x}, x) \quad \text { for all } z \in[x, \tilde{x}] \text {. }
$$

Dually to Proposition 14 we get

Proposition 15. Let $U$ be a proper uninorm having $e$ as neutral element. Let $y<e$ be an idempotent element of $U$. If there exists $x>$ e such that $U(x, y)=$ $\tilde{x} \in] e, x[$ then

$U(z, y)=\tilde{x}$ and $U(z, x)=U(\tilde{x}, x) \quad$ for all $z \in[\tilde{x}, x]$.

Propositions 14 and 15 have the following corollary. 
Corollary 3. Let $U$ be a proper uninorm having e as neutral element.

(i) Assume $x<e$ is an idempotent element of $U$. Then either $x \nVdash_{U} y$ for all $y \in[e, 1]$ or there exists an interval $] a, b] \subset[e, 1]$ such that $x \|_{U} z$ for all $z \in] a, b]$.

(ii) Assume $x>e$ is an idempotent element of $U$. Then either $x \nVdash_{U} y$ for all $y \in[0, e]$ or there exists an interval $\left[a, b\left[\subset[0, e]\right.\right.$ such that $x \|_{U} z$ for all $z \in[a, b[$.

Kalina and Král' [18] introduced uninorms which are strictly increasing on $] 0,1\left[^{2}\right.$, but not continuous. The construction method was further studied in [1, 27]. Since we are not able to distinguish among continuous t-norms $T$ (t-conorms $S$ ) by means of the relation $\preceq_{T}\left(\preceq_{S}\right)$, we are not able to characterize unambiguously uninorms which are strictly increasing on $] 0,1\left[^{2}\right.$. We present the main idea of the construction method, paving, in case the basic 'brick' is the product t-norm $T_{\pi}$ :

(a) we split the interval ]0,1[ into infinitely countably many disjoint right-closed subintervals $\left\{I_{j} ; j \in\right.$ $\mathcal{J}\}$, where $\mathcal{J}$ is an index set and $\left(\mathcal{J}, \circledast, j_{0}\right)$ is a commutative increasing monoid and $j_{0}$ is its neutral element,

(b) $\left.\left.\vartheta_{j}: I_{j} \rightarrow\right] 0,1\right]$ is an increasing bijection.

The resulting uninorm is defined by:

$$
\begin{aligned}
U_{p}(x, y)= & \vartheta_{i \circledast j}^{-1}\left(T_{\pi}\left(\vartheta_{i}(x), \vartheta_{j}(y)\right)\right) \text { for } x \in J_{i}, y \in J_{j} \\
& 0 \quad \text { if } \min \{x, y\}=0 \\
& 1 \quad \text { otherwise. }
\end{aligned}
$$

Concerning the properties of $\preceq_{U_{p}}$ there are two possibilities depending whether $\left(\mathcal{J}, \circledast, j_{0}\right)$ is a group or not.

Proposition 16. Let $U_{p}$ be a uninorm defined by (6), $\left(\mathcal{J}, \circledast, j_{0}\right)$ be a commutative group and $\left\{I_{j} ; j \in \mathcal{J}\right\}$ be a system of disjoint right-closed intervals whose union is $] 0,1[$. Then:

(i) for every $j \in \mathcal{J}$ and all $(x, y) \in I_{j}^{2}$ we have

$$
x \preceq_{U_{p}} y \Leftrightarrow x \leq y,
$$

(ii) for all $i, j \in \mathcal{J}, i \neq j$, all $x \in J_{i}$ and $y \in J_{j}$ we have

$$
\begin{array}{lll}
x \sim_{U_{p}} y & \Leftrightarrow & \vartheta_{j}(y)=\vartheta_{i}(x), \\
x \preceq l_{U_{p}} y & \Leftrightarrow & \vartheta_{i}(x) \leq \vartheta_{j}(y) .
\end{array}
$$

Proposition 17. Let $U_{p}$ be a uninorm defined by (6), $\left(\mathcal{J}, \circledast, j_{0}\right)$ be a commutative monoid without inverse elements, with the neutral element $j_{0}$ and $\left\{I_{j} ; j \in \mathcal{J}\right\}$ be a system of disjoint right-closed intervals whose union is ]0, 1[. Then:

(i) for every $j \in \mathcal{J}$ and all $(x, y) \in I_{j}^{2}$ we have

$$
x \preceq U_{p} y \Leftrightarrow x \leq y,
$$

(ii) for all $i, j \in \mathcal{J}, i \neq j$, all $x \in J_{i}$ and $y \in J_{j}$ we have

$x \preceq_{U_{p}} y \Leftrightarrow \vartheta_{i}(x) \leq \vartheta_{j}(y)$ and $(\exists k \in \mathcal{J})(j \circledast k=$ i),

$x \|_{U_{p}} y$ if and only if one of the following holds

$(\nexists k \in \mathcal{J})(j \circledast k=i \vee i \circledast k=j)$,

$(\exists k \in \mathcal{J})(j \circledast k=i)$ and $\vartheta_{i}(x)>\vartheta_{j}(y)$,

$(\exists k \in \mathcal{J})(i \circledast k=j)$ and $\vartheta_{i}(x)<\vartheta_{j}(y)$.

We could formulate dual theorems to Propositions 16 and 17 for the case when the basic 'brick' is the probabilistic sum t-conorm.

\section{Conclusion}

The results presented in this paper are aimed to characterize classes of uninorms by means of a pre-order the induce. We have succeeded in getting full characterization for uninorms which are continuous on $] 0,1\left[^{2}\right.$, as well as for uninorms with continuous underlying tnorm and t-conorm, and for those which are locally internal on the boundary and on $A(e)$. Further, it is possible to distinguish whether a uninorm is conjunctive or disjunctive. In some other cases we have partial results.

\section{Acknowledgement}

The work of Martin Kalina was supported from the Science and Technology Assistance Agency under contract No. APVV-18-0052, and from the VEGA grant agency, grant No. 2/0069/16 and 1/0006/19.

\section{References}

[1] S. Bodjanova, M. Kalina, Block-wise construction of commutative increasing monoids. Fuzzy Sets and Systems 324 (2017) 91-99.

[2] T. Calvo, A. Kolesárová, M. Komorníková, R. Mesiar, Aggregation operators: Properties, classes and construction methods. In: T. Calvo, G. Mayor, R. Mesiar (Eds.) Aggregation operators, Physica-Verlag Heidelberg, 2002, pp. 3-104.

[3] J. Casasnovas, G. Mayor, Discrete t-norms and operations on extended multisets. Fuzzy Sets and Systems 1599 (2008) 1165-1177. 
[4] A. H. Clifford, Naturally totally ordered commutative semigroups. Amer. J. Math, 76 (1954) 631646.

[5] J. Dombi, Basic concepts for a theory of evaluation: The aggregative operator. European Journal of Operational Research 10 (3) (1982) 282293.

[6] J. Drewniak, P. Drygaś, On a class of uninorms. international Journal of Uncertainty, Fuzziness and Knowledge-Based Systems 10, Suppl. (2002) $5-10$.

[7] P. Drygaś, On the structure of continuous uninorms. Kybernetika 43, 2 (2007) 183-196.

[8] P. Drygaś, On monotonic operations which are locally internal on some subset of their domain. In: Štepnička et al. (Eds.) New Dimensions in Fuzzy Logic and Related Technologies, Proceedings of the 5 th EUSFLAT Conference 2007, Universitas Ostraviensis, vol. II, Ostrava, 2007, 185-191.

[9] P. Drygaś, D. Ruiz-Aguilera, J. Torrens, A characterization of a class of uninorms with continuous underlying operators, Fuzzy Sets and Systems 287 (2016) 137-153.

[10] J. Fodor, B. De Baets, A single-point characterization of representable uninorms. Fuzzy Sets and Systems 202 (2012) 89-99 .

[11] Ü. Ertuğrul, M. N. Kesicouğlu, F. Karaa̧l, Ordering based on uninorms. Information Sciences 330 (2016) 315-327.

[12] J. Fodor, R. R. Yager, A. Rybalov, Structure of uninorms. International Journal of Uncertainty, Fuzziness and Knowledge-based Systems 5 (1997) 411-422.

[13] R. Hartwig, How to partially order regular elements, Math. Japon. 25 (1980) 1-13.

[14] D. Hliněná, M. Kalina, P. Král', Nonrepresentable uninorms. In: EUROFUSE 2013, Uncertainty and Imprecision Modelling in Decision Making. Servicio de Publicaciones de la Universidad de Oviedo, Oviedo, 2013, 131-138.

[15] D. Hliněná, M. Kalina, P. Král', A class of implications related to Yager's $f$-implications, Information Sciences 260 (2014) 171-184.

[16] D. Hliněná, M. Kalina, P. Král', Pre-orders and orders generated by uninorms. In: 15th Int. Conference IPMU 2014, Proceedings, Part III, Montpellier, France, Springer, Heidelberg, 2014, 307-316 .
[17] S. Hu, Z. Li, The structure of continuous uninorms. Fuzzy Sets and Systems, 124 (2001) 4352.

[18] M. Kalina, P. Král', Construction of commutative associative operations by paving. In: J. M. Alonso, H. Bustince, Reformat (eds.) IFSAEUSFLAT 2015, Atlantis Press, Gijón, 2015, 1201-1207.

[19] F. Karaçal, M. N. Kesicioğlu, A t-partial order obtained from t-norms. Kybernetika 47, No. 2 (2011) 300-314.

[20] E. P. Klement, R. Mesiar, E. Pap, Triangular Norms. Springer, Berlin, Heidelberg, 2000

[21] J. Martín, G. Mayor, J. Torrens, on locally internal monotonic operations, Fuzzy Sets and Systems 137 (2003), 27-42.

[22] H. Mitsch, A natural partial order for semigroups. Proc. AMS 97,3 (1986) 384-388.

[23] K. Nambooripad, The natural partial order on a regular semigroup, Proc. Edinburgh Math. Soc. 23 (1980) 249-260.

[24] M. Petrík, R. Mesiar, On the structure of special classes of uninorms. Fuzzy sets and Systems, 240 (2014) 22-38.

[25] D. Ruiz-Aguilera, J. Torrens, B. De Baets, J. Fodor, Some remarks on the characterization of idempotent uninoms. In: E. Hüllermeier, R. Kruse, F. Hoffmann (Eds.), Proc. 13th IPMU 2010 Conference on Computational Intelligence for Knowledge-Based Systems Design, LNAI, vol. 6178, Springer, Berlin, Heidelberg, 2010, pp. 425434.

[26] R. R. Yager, A. Rybalov, Uninorm aggregation operators. Fuzzy Sets and Systems 80 (1996) 111120.

[27] W. Zong, Y. Su, H. W. Liu, B. De Baets, On the Construction of Associative, Commutative and Increasing Operations by Paving. In: Torra V., Mesiar R., Baets B. (eds) Aggregation Functions in Theory and in Practice. AGOP 2017. Advances in Intelligent Systems and Computing, vol 581. Springer, Cham, 229-240. 